\title{
Phase field modeling of damage in glassy polymers
}

Yuesong Xie ${ }^{1}$, Oleksandr G. Kravchenko른 R. Byron Pipes ${ }^{2}$ and Marisol Koslowski ${ }^{1}$

${ }^{1}$ School of Mechanical Engineering, Purdue University, West Lafayette IN 47907

2 School of Aeronautics and Astronautics, Purdue University, West Lafayette IN 47907

\section{Abstract}

Failure mechanisms in amorphous polymers are usually separated into two types, shear yielding and crazing due to the differences in the yield surface. Experiments show that the yield surface follows a pressure modified von Mises relation for shear yielding but this relation does not hold during crazing failure. In the past different yield conditions were used to represent each type of failure. Here, we show that the same damage model can be used to study failure under shear yielding and crazing conditions. The simulations show that different yield surfaces are obtained for craze and shear yielding if the microstructure is included explicitly in the simulations. In particular the breakdown of the pressure modified von Mises relation during crazing can be related to the presence of voids and other defects in the sample.

\section{Introduction}

Extensive experimental evidence shows that failure of glassy polymers is influenced by deviatoric and volumetric components of deformation. Due to this claim the most widely used yield criterion is a pressure-modified von Mises condition of the form:

${ }^{d e v}={ }_{c r i t} \quad{ }_{v M}{ }^{v o l}$

where $\sigma_{\text {crit }}$ is a constant representing the stress required for yielding under pure shear stress and $\mu_{v M}$ is a coefficient that describes the dependence of the deviatoric stress on the volumetric stress at failure. This coefficient is temperature dependent and it is in the range 0.3 to $1^{1}$. The volumetric stress and deviatoric stress in Equation (1) can be written in terms of the principal stresses as:

$$
{ }^{v o l}=\frac{{ }_{11}+{ }_{22}+{ }_{33}}{3}
$$




$$
d e v=\sqrt{\frac{1}{2}\left[\begin{array}{ll}
\left(\begin{array}{ll}
11 & 22
\end{array}\right)^{2}+\left(\begin{array}{ll}
22 & 33
\end{array}\right)^{2}+\left(\begin{array}{ll}
33 & 11
\end{array}\right)^{2}
\end{array}\right]}
$$

Figure 1 shows yield surfaces for Polymethyl methacrylate (PMMA) from the experimental work of Quinson et al. ${ }^{1}$ and Bowden et al. ${ }^{2}$. Quinson et al. ${ }^{1}$ tested three different loading conditions at room temperature: uniaxial compression, plane strain compression and simple shear. The onset of yielding is determined by these authors when the maximum loading stress with zero residual strain is reached. Bowden et $a l^{2}$ investigated plane strain compression of thin sheets of PMMA at room temperature. The yield condition in this case is determined at the maximum compressive stress. These yield surfaces correspond to shear and compressive stresses conditions and follow the pressure-modified von Mises condition in Equation 1.

The experiments of Sternstein et al. ${ }^{3}$ and Argon et al.4,5 examined the behavior under tensile loading. Under this condition zones of fibrillation or crazes develop that result in brittle failure of the polymer. The onset of crazing observed in these experiments is also shown in Figure 1. The data of Sternstein et al. ${ }^{6}$ is obtained for PMMA and the experiment of Argon et al. ${ }^{4}$ is for polystyrene. The results of Argon et $a l^{4}$, correspond to the applied stress at the onset of crazing while in a later work Argon $^{5}$ calculated the local values of stress from a model that takes into account initial surface grooves. It is clear that under positive volumetric stress the yield surface in some experiments do not follow Equation 1.

Due to its engineering impact, polymer damage has been the focus of extensive modeling efforts. A number of computational models, ranging from atomistic 7,8 to continuum 9-13, have been utilized to study polymer failure as well as polymer composite materials ${ }^{14-16}$. While atomistic simulations give insight information into the material behavior, a connection to experimental results remains elusive due to the differences in time and length scales. At the continuum level new mathematical approaches to failure are being developed in which the damage is characterized by a single material parameter that can be calibrated with experiments or atomistic simulations. In these theories a phase field variable indicates how damage is extended over the domain and its evolution 17-21. A difference with previous approaches such as cohesive zone models is that the damage is not limited to a two dimensional manifold but it is defined over a finite volume which makes the phase field method advantageous for crazing and shear yielding where diffuse damage zones are observed 1 . 


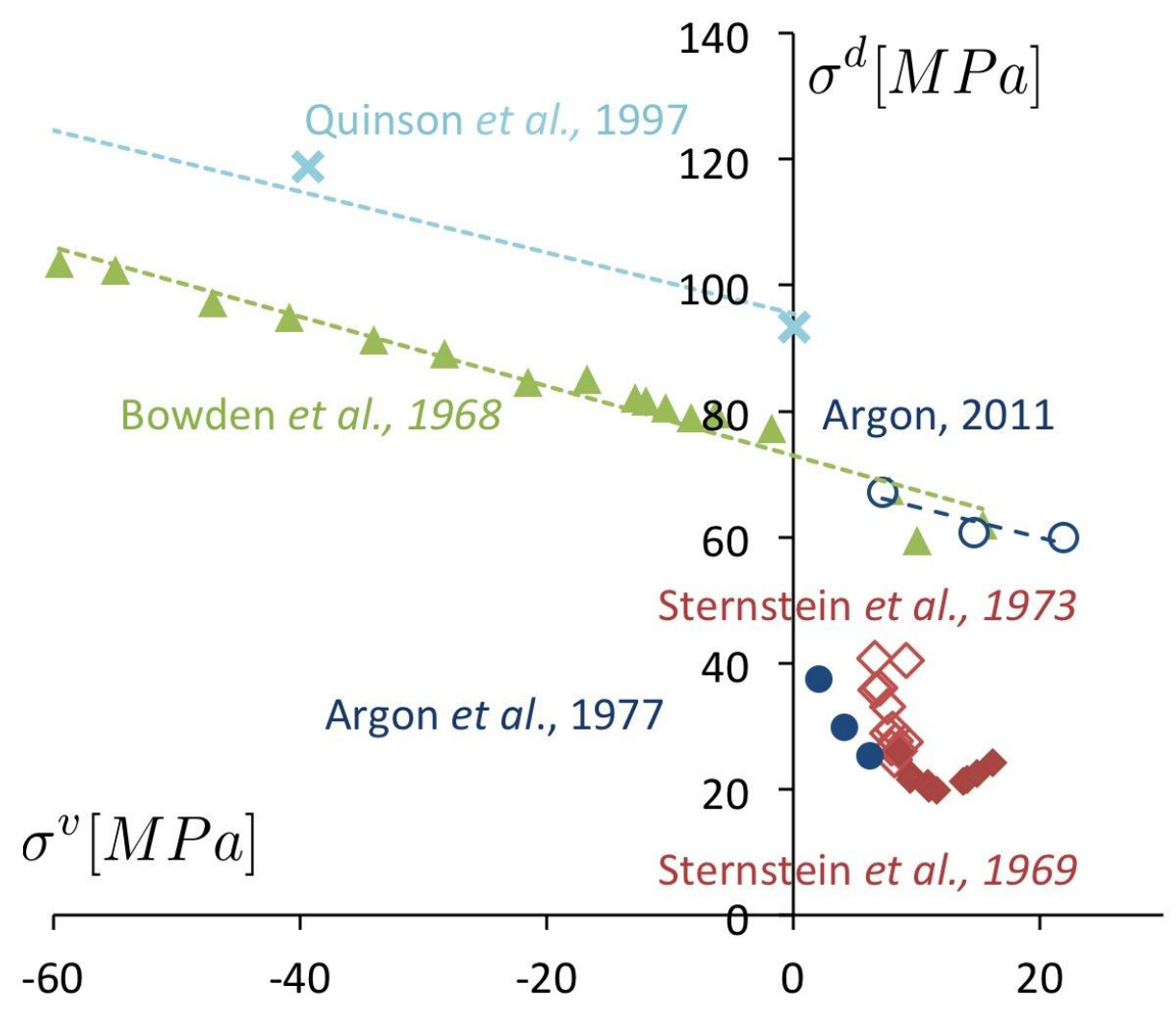

Figure 1: Yield surfaces for PMMA extracted from the experiments of Quinson et al. ${ }^{1}$, Bowden et al. ${ }^{2}$ (green triangles), Sternstein et al. ${ }^{6}$ (solid red diamonds), Sternstein et al. ${ }^{3}$ (open red diamonds), and for polystyrene from the experiments of Argon et al. ${ }^{4}$ (solid blue circles) and Argon ${ }^{5}$ (open blue circles).

In this paper, numerical simulations with a phase field damage model (PFDM) are performed to study the onset of yielding in amorphous polymers. Two different brittle damage models that separate volumetric and deviatoric contributions are considered in the PFDM. In the first model, introduced by Amor et al. ${ }^{18}$ only the dilatational and the deviatoric part produce damage. The second model, developed by Miehe et al. ${ }^{19}$, takes into account the sign of the principal strain directions. The predicted yield surfaces show that to obtain hardening in compression it is necessary to consider the sign of the individual principal strain directions and therefore, the second model is adopted. The parameters of this model are calibrated with experiments performed in a simple tension dog bone and a single edge notch tension (SENT) PMMA specimen. The calibrated model is used to reproduce the loading conditions in the experiments in Figure 1. The simulations show that the same PFDM model can be used to predict the experimental results in Figure 1. 
Including voids in the sample explains the difference in the behavior under positive volumetric stress during the onset of crazing.

The paper is organized as follows, in Section 2 the PFDM is presented. The PFDM is calibrated against two experiments in Section 3. The calibrated model is used to predict the behavior of PMMA in different loading conditions and geometries. A summary of the results is in Section 4.

\section{Phase Field Damage Model formulation}

In the PFDM local damage is represented by a field variable. The original approach was a variational formulation introduced by Francfort and Marigo ${ }^{17}$. Later, this model was extended to a phase field implementation to study quasi-static and dynamic brittle failure with different material responses and loading conditions 18,19,22-26. Following Griffith's criterion the energy required to create a crack of area $\Gamma$ is:

$$
W_{f}[]=\int G_{c} d x
$$

where $G_{c}$ is the fracture toughness, i.e., the energy density necessary to create a unit surface crack. In the phase field approach 27 the crack surface is parameterized by a smooth representation as shown in Figure 1. Therefore, the integral in the crack surface is replaced by the following :

$$
\int_{\Gamma} G_{c} d x \simeq \int_{V} G_{c}\left(\frac{d^{2}}{4 l_{0}}+l_{0}|\nabla d|^{2}\right) d x
$$

where $d(x) \in[0,1]$ is the phase field that tracks damage in the material with $d=1$ representing a crack and $d=0$ undamaged material. The length scale parameter $l_{0}$ governs the regularization of the damaged region. As $l_{0} \rightarrow 0$, the volume integral converges to the original expression in Equation (4). 

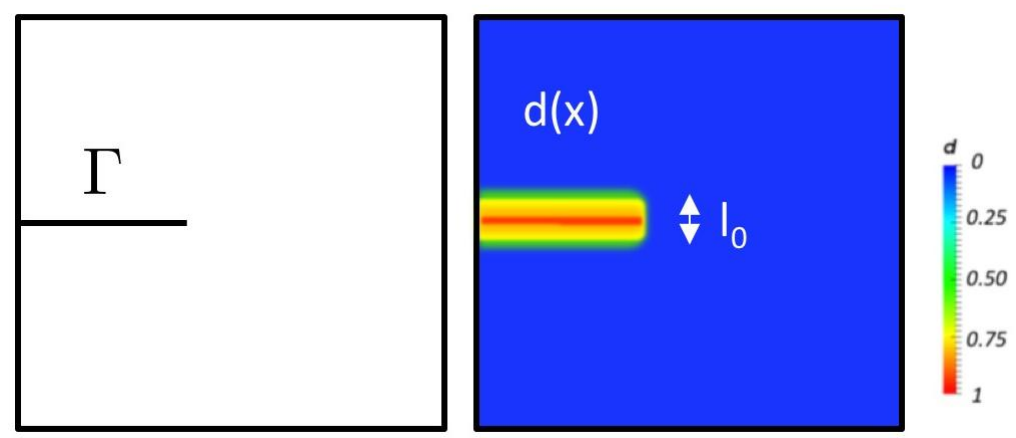

Figure 2: (a) Schematic representation of a solid with a crack. (b) Approximation of the crack with the phase field $d(x)$.

The elastic energy is decomposed into two terms ${ }^{17}$, one due to compression, $W_{e}^{(-)}$, and a second term due to tension $W_{e}^{(+)}$:

$$
W_{e}[, d]=W_{e}^{(+)}[, d]+W_{e}^{()}[]
$$

Assuming that damage occurs in tension $W_{e}^{(+)}[\varepsilon, d]$ is the part of elastic energy that is degraded by damage and it is of the form

$W_{e}^{(+)}[, d]=\int_{V}\left(\begin{array}{ll}1 & d\end{array}\right)^{2} a^{+}[] d x$

The monotonically increasing function $(1-d)^{2}$ describes the loss of stiffness of the damaged materials while $W_{e}^{(-)}[\varepsilon]$ is not affected by damage $W_{e}^{()}[]=\int_{V} a[] d x$

where $a^{+}[\varepsilon]$ and $a^{-}[\varepsilon]$ are strain energy densities. Different forms of these strain energies will be defined in Sections 2.2 and 2.3. The elastic strain energy rate can be obtained as:

$$
\dot{W}_{e}[\varepsilon, \dot{\varepsilon}, d, \dot{d}]=\int_{V}\left(\sigma_{i j}: \dot{\varepsilon}_{i j}-2(1-d) a^{+}[\varepsilon] \dot{d}\right) d x
$$

where

$$
{ }_{i j}=\left(\begin{array}{ll}
1 & d
\end{array}\right)^{2} \frac{a^{+}[]_{i j}}{{ }_{i j}}+\frac{a[]_{i j}}{{ }_{i j}}
$$

and the energy dissipation due to crack formation follows as

$$
\dot{W}_{f}[d, \dot{d}]=\int_{V} G_{c}\left(\frac{d}{2 l_{0}}+2 l_{0} \Delta d\right) \dot{d} d x
$$

where $\Delta$ is the Laplacian. Due to the irreversibility nature of the damage, the energy dissipation in Equation (11) should be positive. This constrain is satisfied by imposing the following two local conditions: 


$$
\begin{gathered}
\frac{d}{2 l_{0}}+2 l_{0} \Delta d \geq 0 \\
\dot{d} \geq 0
\end{gathered}
$$

In order to satisfy the conditions in Equation (12), an extended Lagrangian is defined 19

$$
L[\varepsilon, \dot{\varepsilon}, d, \dot{d}, \lambda]=\dot{W}_{e}[\varepsilon, \dot{\varepsilon}, d, \dot{d}]+D[d, \dot{d}, \lambda]
$$

where $D[d, \dot{d}, \lambda]$ is the extended dissipation functional

$$
D[d, \dot{d}, \lambda]=\int_{V}\left(2(1-d) a^{+}[\varepsilon] \dot{d}-\lambda Y_{c r}\right) d x \geq 0
$$

where $\lambda$ is a Kuhn-Tucker multiplier and $Y_{c r}$ is an inequality constraint function defined as

$$
Y_{c r}=2(1 \quad d) a^{+}[] G_{c}\left(\frac{d}{2 l_{0}}+2 l_{0} d\right) \leq 0
$$

The variation of the extended Lagrangian with respect to the field variables yields the following Kuhn-Tucker equations

$$
\begin{gathered}
\sigma_{i j, j}=0 \\
\dot{d}=\lambda \geq 0 \\
Y_{c r} \leq 0 \\
\dot{d} \cdot Y_{c r}=0
\end{gathered}
$$

The first equation in (16) is the equilibrium condition. The second equation enforces that the damage can only increase. The last three equations in (16) together with Equation (15) lead to the governing equation of the phase field.

$$
\left(\frac{4 l_{0} a^{+}[]}{G_{c}}+1\right)(1 d) 4 l_{0}^{2} \frac{\partial^{2} d}{\partial x_{i} \partial x_{i}}=1
$$

\subsection{Alternate minimization algorithm}

An alternate minimization algorithm is used to optimize the energy with the local minima ${ }^{18,27}$. This algorithm consists of solving a series of minimization of subproblems ${ }^{28}$. At each loading step, the displacement $\boldsymbol{u}$ is first solved at a fixed phase field $d$. Then $d$ is solved at fixed $\mathbf{u}$ with the irreversibility condition. This process repeats until the convergence is reached, i.e., the change in the variable between two iterative steps is less than certain tolerance. For instance, given the solution on displacements and damage $\left(\boldsymbol{u}_{i-1}, d_{i-1}\right)$ at a loading step $t_{i-1}$, the solution $\left(\boldsymbol{u}_{i}\right.$ $\left.d_{i}\right)$ at $t_{i}$ is obtained as follows:

1) $\operatorname{Set}\left(\boldsymbol{u}^{(0)}, d^{(0)}\right)=\left(\boldsymbol{u}_{i-1}, d_{i-1}\right)$ 
2) Set $p=1$.

a. Solve the predictor problem $\boldsymbol{u}^{(p)}$ with $d=d^{(p-1)}$

b. Solve Equation for $d^{(p)}$ with $\boldsymbol{u}=\boldsymbol{u}^{(p)}$ under the constraint $d \geq d_{i-1}$

c. Repeat until $\left\|d^{(p)}-d^{(p-1)}\right\| \leq \delta$ or $p=p+1$

3) Set $\left(\boldsymbol{u}_{i}, d_{i}\right)=\left(\mathbf{u}^{(p)}, d^{(p)}\right)$

The simulations are performed using a 3D parallel software, MEMOSA, which is based on the cell-centered finite-volume method ${ }^{28}$. In the following sections two strain energy densities are analyzed.

\subsection{Dilatational and shear strain driven damage}

The pressure modified von Mises relation reflects that the onset of damage takes place at lower deviatoric stress when polymers are under dilatational stress than when they are under compression. In order to reproduce this material behavior, in the model proposed by Amor et al. ${ }^{18}$ the strain energy density is decoupled into volumetric and deviatoric parts as follows:

$$
\begin{aligned}
& a^{+\mathrm{A}}\left[\mathrm{]}=\frac{1}{2}\left(+\frac{2}{3}\right)\left\langle v^{2}+\operatorname{tr}\left[\left(\begin{array}{l}
d e v \\
i j
\end{array}\right)^{2}\right]\right.\right. \\
& a^{\mathrm{A}}[\mathrm{]}]=\frac{1}{2}\left(+\frac{2}{3}\right)(v\langle v\rangle)^{2}
\end{aligned}
$$

where $\lambda$ and $\mu$ are the Lamé constants, and

$$
\langle x\rangle=\left\{\begin{array}{l}
x \text { if } x \geq 0 \\
0 \text { if } x<0
\end{array}\right.
$$

\subsection{Dilatational and positive principal strain directions driven damage}

Miehe et al. ${ }^{19}$ formulated a different approach to decouple the volumetric and deviatoric parts of the deformation based on the spectral decomposition of the strain tensor. The tensor $\Lambda$ that contains the principal values of the strain tensor, can be expressed as

$$
\varepsilon=P \Lambda P^{T}
$$

where $P$ are the orthogonal eigenvectors of the strain tensor. $\Lambda$ is further decomposed as:

$$
\Lambda^{-}=\Lambda-\Lambda^{+}
$$


with:

$$
\Lambda^{+}=\left[\begin{array}{ccc}
\left\langle\lambda_{1}\right\rangle & 0 & 0 \\
0 & \left\langle\lambda_{2}\right\rangle & 0 \\
0 & 0 & \left\langle\lambda_{3}\right\rangle
\end{array}\right]
$$

Then, the strain energy densities $a^{+M}[\varepsilon]$ and $a^{-M}[\varepsilon]$ are defined as

$$
\begin{aligned}
& a^{+\mathrm{M}}\left[\mathrm{]}=\frac{1}{2}\left\langle v^{v}\right\rangle^{2}+\operatorname{tr}\left[\left(\begin{array}{c}
+ \\
i j
\end{array}\right)^{2}\right]\right. \\
& a^{\mathrm{M}}[\mathrm{]}]=\frac{1}{2}\left({ }^{v}\left\langle{ }^{v}\right\rangle\right)^{2}+\operatorname{tr}\left[\left({ }_{i j}\right)^{2}\right]
\end{aligned}
$$

where $\varepsilon_{i j}^{+}=P \Lambda^{+} P^{T}$ and $\varepsilon_{i j}^{-}=P \Lambda^{-} P^{T}$. The similarity between the energy density expressions proposed by Miehe et al. ${ }^{19}$ and Amor et al. ${ }^{18}$ is that the compressive part of the strain does not contribute to damage producing an asymmetric response in tension and compression.

\subsection{Homogeneous uniaxial loading}

The distinct responses of the two material constitutive models presented in Sections 2.1 and 2.2 are analyzed in this section in a homogeneous sample under an applied strain $\varepsilon_{11}$. The stress components in the other two directions are set to zero. Under a homogeneous deformation assumption the evolution of the phase field, Equation (17), simplifies to:

$$
\left(\frac{4 l_{0} a^{+}[]}{G_{c}}+1\right)(1 \quad d)=1
$$

In the model of Amor et al. ${ }^{18}$, the stress components can be obtained from Equation (10) and (18) as:

$$
{ }_{i j}[, d]=\left(+\frac{2}{3}\right)\left({ }^{v}\left\langle v^{v}\right\rangle\right)+\left(\begin{array}{ll}
1 & d
\end{array}\right)^{2}\left(\left(\begin{array}{l}
3 \\
3
\end{array}\right)\left\langle v^{v}\right\rangle+2 \begin{array}{l}
\text { dev } \\
i j
\end{array}\right)
$$

Under the current loading conditions the other strain components are related to $\varepsilon_{11}$ by the Poisson ratio as

$$
{ }_{22}={ }_{33}=\quad{ }_{11}
$$

Therefore, the volumetric strain $\varepsilon^{v}=(1-2 v) \varepsilon_{11}$ is positive and the traction free conditions lead to 


$$
\begin{aligned}
& { }_{22}=\left(\begin{array}{ll}
1 & d
\end{array}\right)^{2}\left(\left(+\frac{2}{3}\right)^{v}+2 \begin{array}{c}
d e v \\
22
\end{array}\right)=0 \\
& { }_{33}=\left(\begin{array}{ll}
1 & d
\end{array}\right)^{2}\left(\left(\begin{array}{l}
+\frac{2}{3}
\end{array}\right)^{v}+2 \begin{array}{c}
\text { dev } \\
33
\end{array}\right)=0
\end{aligned}
$$

The solution of the phase field can be obtained from Equations (24) and (26) as:

$$
d=\frac{(1+)_{11}^{2}}{(1+)_{11}^{2}+\frac{G_{c}}{4 l_{0}}}
$$

And the stress is

$$
{ }_{11}=\left(\frac{(1+)}{G_{c} / 4 l_{0}}\left({ }_{11}\right)^{2}+1\right)^{2} \cdot 2(1+)_{11}
$$

In the model of Miehe et al. ${ }^{19}$ using the the strain energy densities in Equation (23) the stress components result:

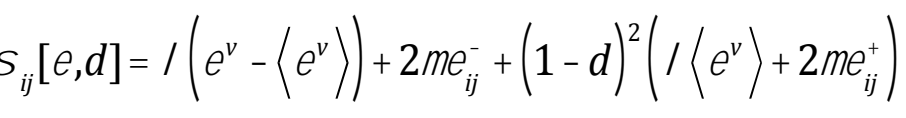

Similarly, $\varepsilon^{v} \geq 0$ and the traction free boundary condition results in the following conditions:

$$
\begin{aligned}
& { }_{22}=\left(\begin{array}{lll}
1 & d
\end{array}\right)^{2}{ }^{v}+2{ }_{22}=0 \\
& { }_{33}=\left(\begin{array}{lll}
1 & d
\end{array}\right)^{2}{ }^{v}+2{ }_{33}=0
\end{aligned}
$$

and the strain components are

$$
{ }_{22}={ }_{33}=\frac{(1 d)^{2}}{2\left(\left(\begin{array}{ll}
1 & d
\end{array}\right)^{2}+\right)^{11}}
$$

Using Equations (24) and (32) the following expression for the damage field is obtained 


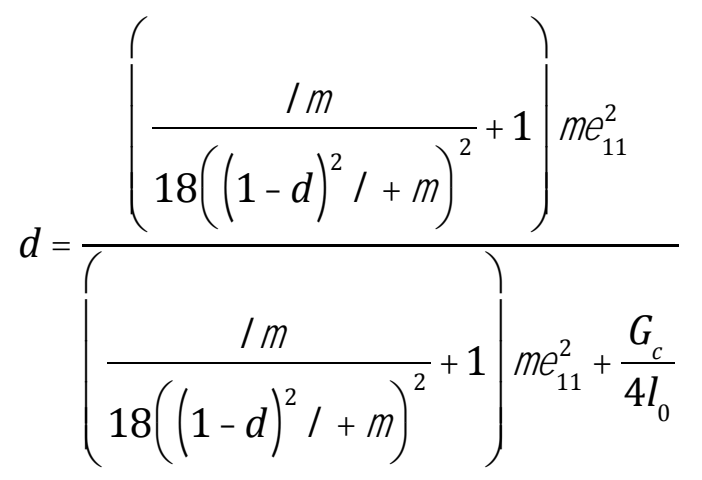

The non-linear Equation (33) can be solved numerically and the axial stress is obtained as

$$
{ }_{11}=\left(\begin{array}{ll}
1 & d
\end{array}\right)^{2} \times{ }^{v}+\left(\begin{array}{ll}
1 & d
\end{array}\right)^{2} \times_{2}{ }_{11}
$$

The stress strain curves and the evolution of the damage field as a function of $\varepsilon_{11}$ for both models are compared in Figure 2. The material properties used are $\lambda=2407 \mathrm{MPa}, \mu=1240 \mathrm{MPa}$ and $G_{c} / 4 l_{0}=45 \mathrm{MPa}$ following the previous work of Xie et al. ${ }^{28}$.

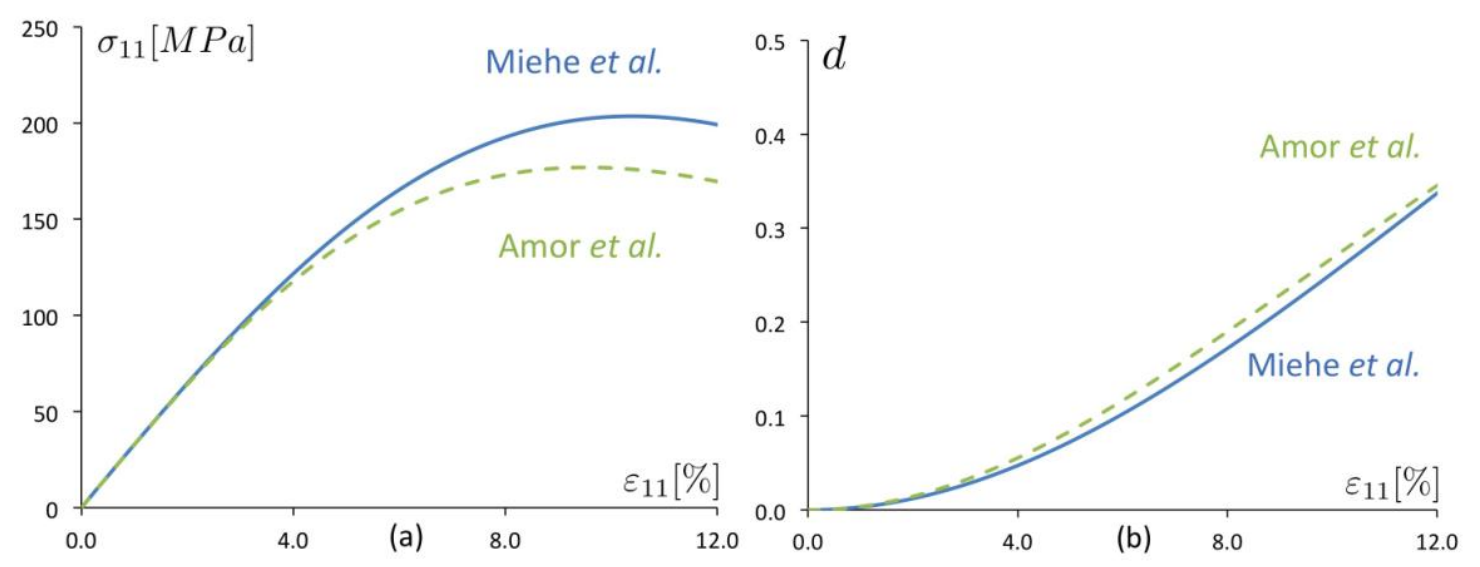

Figure 3: (a) Stress-strain curves and (b) damage field evolution for uniaxial loading using Miehe et al. ${ }^{19}$ and Amor et al. ${ }^{18}$ constitutive models.

\subsection{Onset of yielding}


The onset of yielding in this work is determined following Xie et al. ${ }^{28}$ and Jaramillo et al. ${ }^{8}$ and it is defined as the maximum of the rate of mechanical work per unit volume, $\dot{W}_{\text {mech }}$,

$$
\dot{W}_{\text {mech }}=\sigma_{i j} \dot{\varepsilon}_{i j}
$$

To illustrate the key underlying differences between the two material constitutive models described in Sections 2.1 and 2.2 a 3D strain of the form

$$
\boldsymbol{\varepsilon}=\varepsilon_{11}(t) \cdot\left[\begin{array}{lll}
1 & 0 & 0 \\
0 & \alpha & 0 \\
0 & 0 & \alpha
\end{array}\right]
$$

is applied. The parameter $\alpha \in[-0.5,1]$ takes into account the effect of the triaxiality of the deformation. The stress and strain components are calculated following the same procedure outlined in Section 2.4. The volumetric stress versus the volumetric strain and the deviatoric stress versus the deviatoric strain for $\alpha=0.50,0.00,-0.16$, and -0.33 are shown in Figure 4. It can be noted that for $\alpha \geq 0$ both models agree.
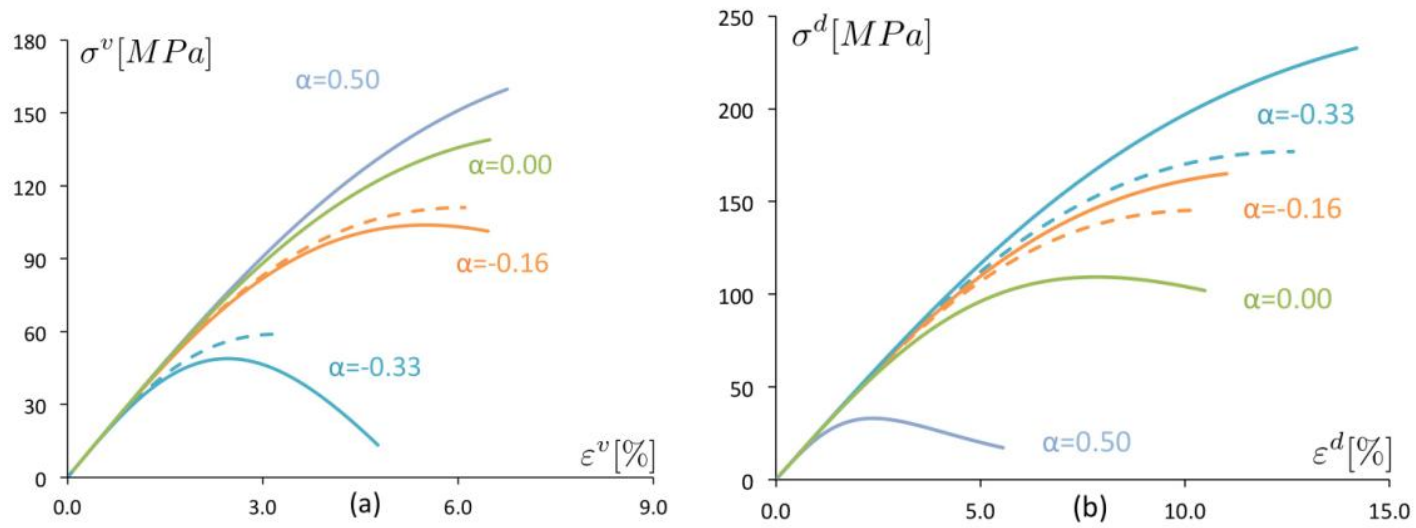

Figure 4: (a) Volumetric stress versus volumetric strain and (b) deviatoric stress versus deviatoric strain for various loading parameter $\alpha$ with Amor $e t$ al. ${ }^{18}$ model (dashed lines) and Miehe et al. ${ }^{19}$ model (solid lines).

Figure 4 shows the rate of mechanical work per unit volume calculated with Equation (35) normalized by the strain rate for both models for $\alpha=0.50,0.00$, 0.16 , and -0.33 . The onset of yielding is defined as the maximum of the curves shown in Figure 4. 


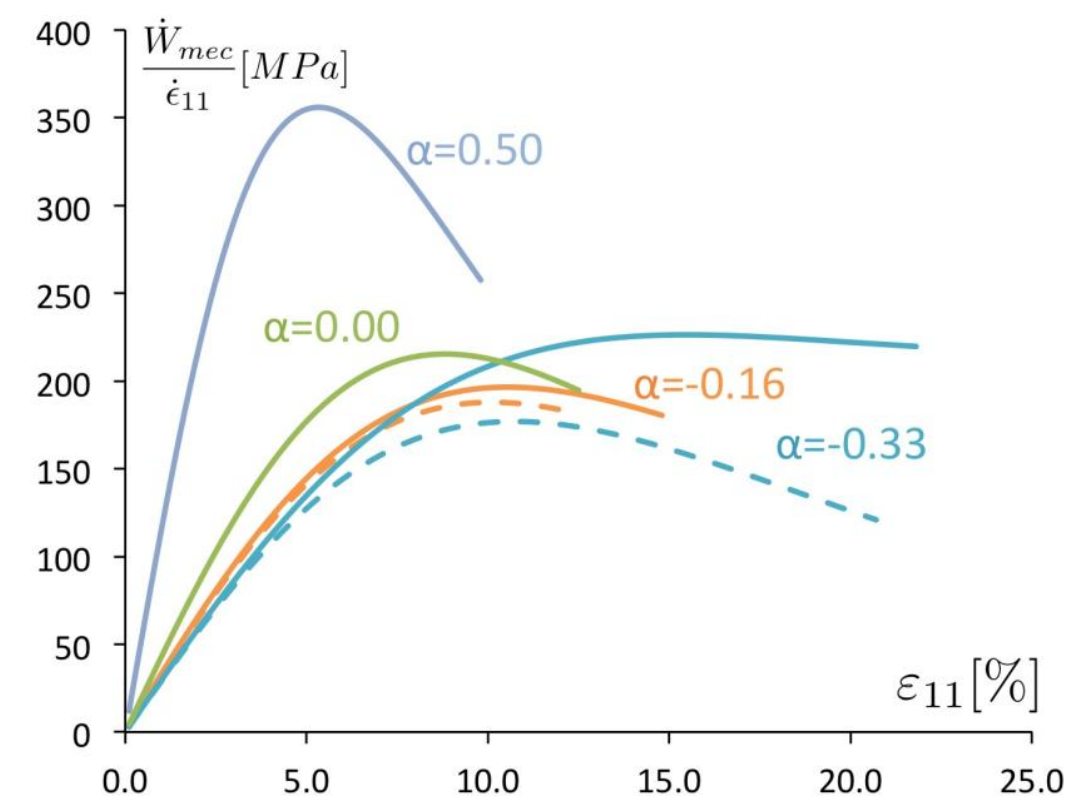

Figure 5: Rate of mechanical work per unit volume normalized by the strain rate for different loading conditions with Amor et al. ${ }^{18}$ model (dashed lines) and Miehe et al. ${ }^{19}$ model (solid lines).

The stress invariants at yield calculated from the data in Figure 4 are shown in Figure 5, in addition the yield surface from molecular dynamics (MD) simulations by Jaramillo et al. ${ }^{8}$ is shown. The calibration of the PFDM model against the MD simulations renders $\mathrm{G}_{\mathrm{C}} / 4 \mathrm{l}_{0}=45 \mathrm{MPa}$ for Amor et al. ${ }^{18}$ model, the same $\mathrm{G}_{\mathrm{C}} / 4 \mathrm{l}_{0}$ is used for Miehe et al. ${ }^{19}$ model. The yield surface obtained with the PFDM based on the model of Miehe et al. ${ }^{19}$ shows an increase in the deviatoric stress at yield in better agreement with the atomistic simulations when the volumetric stress is compressive. Therefore, in the following sections of the paper, this constitutive model described in Section 2.3 is adopted in the simulations. 


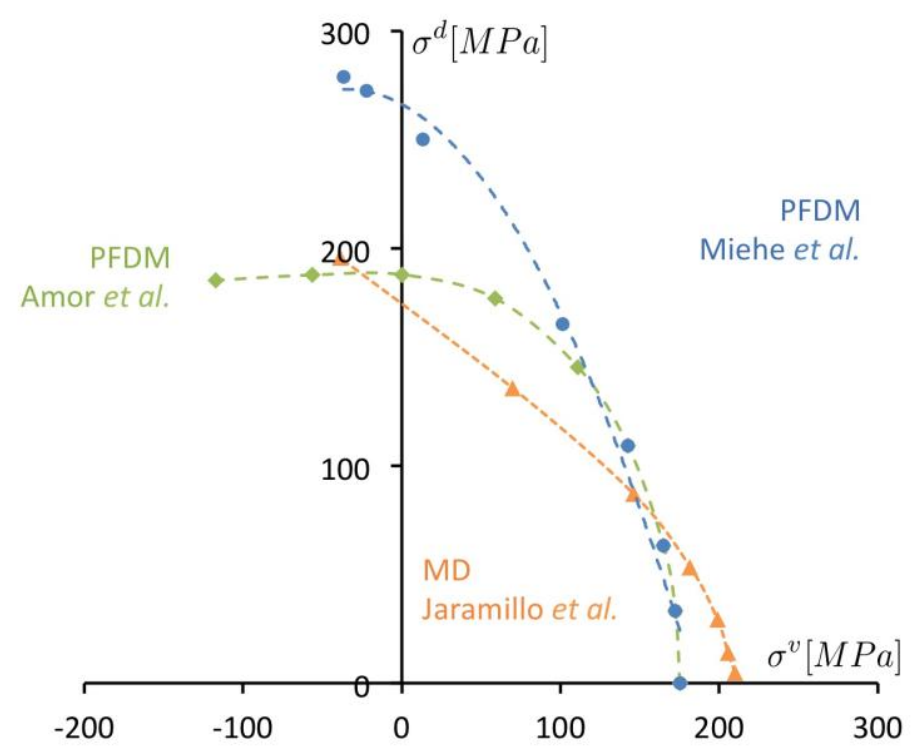

Figure 6: Yield surfaces of a homogeneous sample obtained with Miehe et al. 19 and Amor et al. ${ }^{18}$ constitutive models and Jaramillo et al. ${ }^{8}$ MD simulation.

\section{Damage model calibration}

The calibration of the PFDM against atomistic simulations renders values of yield stress larger than those observed experimentally in PMMA ${ }^{1,2}$ due to the high strain rates used in atomistic simulations and the lack of localization compared to experimental results. Therefore, in this section two sets of experiments are performed to determine the parameters $G_{c}$ and $l_{0}$ for PMMA. The parameter $l_{0}$ in the PFDM model controls the damage width that approximates the crack geometry, whereas the fracture toughness of the material $G_{C}$ is regarded as a true material property.

\subsection{Experimental procedures and sample preparation}

Two groups of PMMA samples were manufactured, a simple tension dog bone specimen and a single edge notch tension (SENT) specimen. PMMA is an amorphous thermoplastic that exhibits brittle fracture behavior under temperatures below $85^{\circ} \mathrm{C}^{29}$. Both types of specimen are cut from a PMMA sheet using a water jet machine.

For the SENT sample an edge notch cut is performed with the water jet and a sharp razor blade is then inserted in the notch and indented to create a sharp precrack ${ }^{30}$ as shown in Figure 6. Aluminum tabs are attached to both ends of the SENT sample using adhesive paste. Figure 7 shows the samples size and geometry. Both 
samples were tested quasi-statically on a standard servo hydraulic MTS machine in displacement control with a cross-head speed of $2 \mathrm{~mm} / \mathrm{min}$ for the dog-bone sample and $0.25 \mathrm{~mm} / \mathrm{min}$ for the SENT sample. Force and displacement data were recorded during the experiment. For the dog-bone specimen a strain gauge rosette was attached to record the longitudinal and transverse strain in.

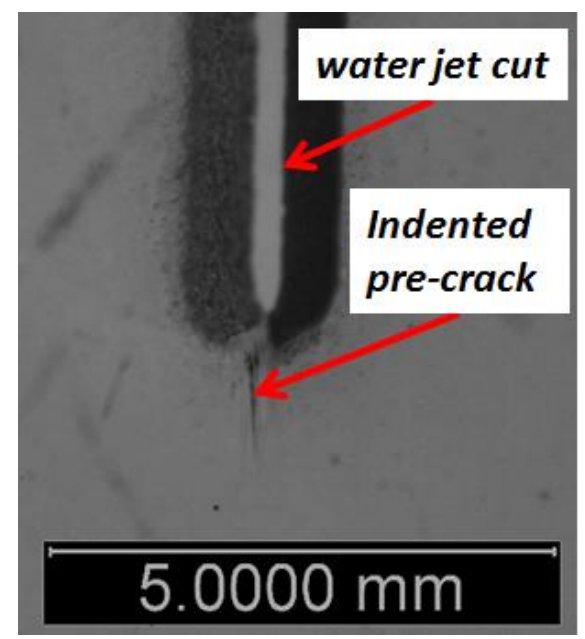

Figure 7: Pre-cracking SENT sample

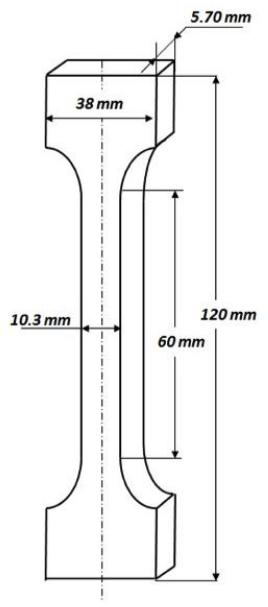

(a)

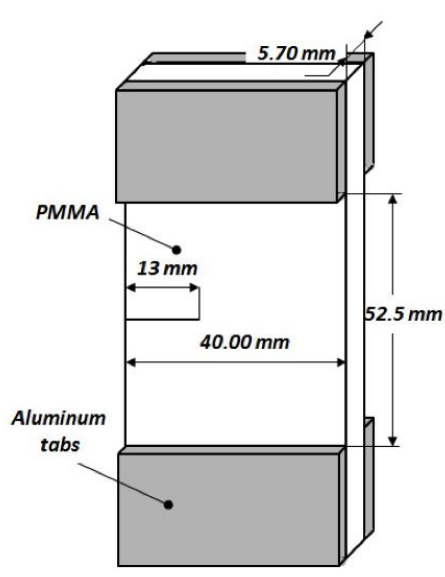

(b)

Figure 7: Sample geometry: (a) Dog-bone sample and (b) SENT sample.

\subsection{Damage model calibration}


The calibration is performed by adjusting the parameters $l_{0}$ and $G_{C}$ to reproduce the experimental stress-strain curves in a dog bone specimen and a SENT sample. The experimental results and simulations are described in the following two sections.

\subsubsection{Dog-bone test}

The experimental results for the dog-bone revealed non-linear behavior in the material before failure occurred at around $44 \mathrm{MPa}$. The Young's modulus and the Poisson's ratio of PMMA are found to be $\mathrm{E}=2483 \mathrm{MPa}$ and $v=0.33$. In this case the state of stress can be considered homogeneous away from the grips and this allows the assumption of a homogeneous damage field in the PFDM simulations. Under this condition, the damage equation from the model of Miehe et al. ${ }^{19}$ reduces to Equation (33) which only depends on the ratio of the two independent model parameters $G_{c}$ and $l_{0}$.

Figure 8 shows that the PFDM simulations with uniaxial loading match the experimental results (using least squares fit) when a ratio $G_{c} /\left(4 l_{0}\right)=2.9 \mathrm{MPa}$ is used in the simulations. It can be noticed that in the experiment the sample breaks before reaching the maximum stress predicted by the PFDM and at lower strain values which can be explained by to the presence of defects in the material that change locally the state of stress leading to premature failure.

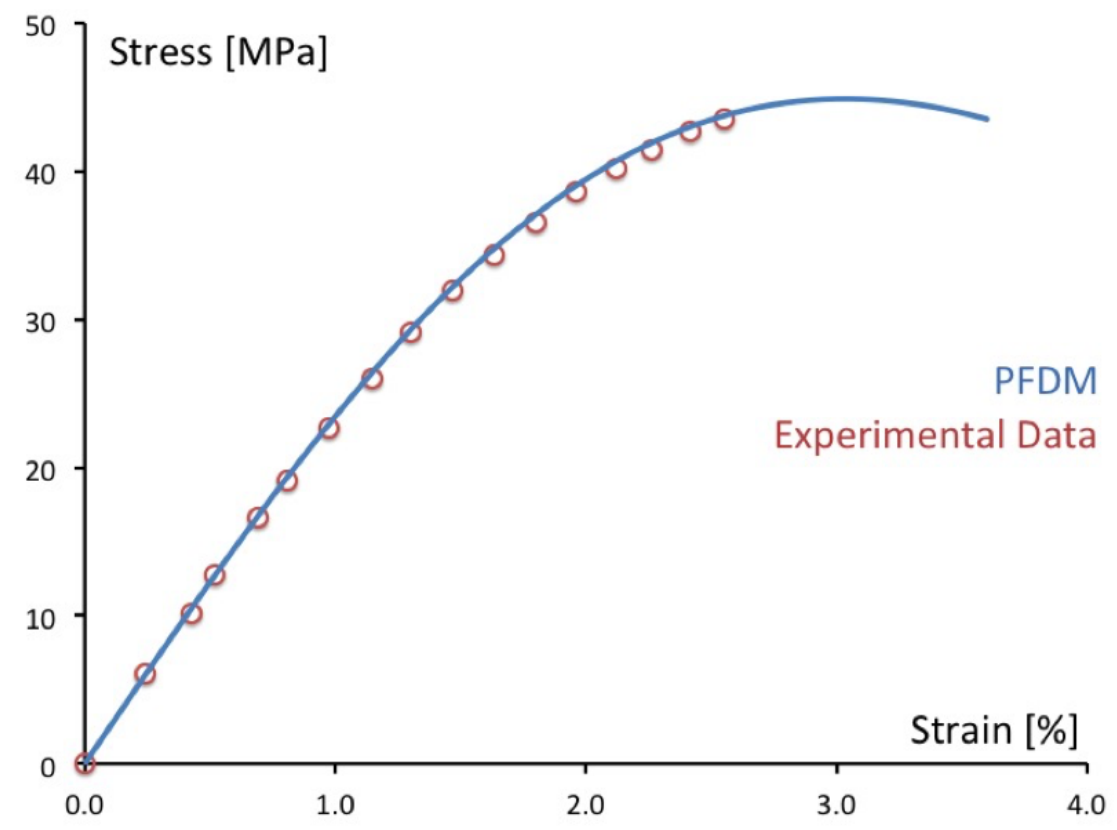

Figure 8: Tensile stress versus tension strain from the dog bone experiment and the fitting curve using PFDM with $\mathrm{G}_{\mathrm{c}} / 4 \mathrm{l}_{0}=2.9 \mathrm{MPa}$. 


\subsubsection{SENT test}

In the SENT test the damage field is not homogeneous due to the presence of the edge notch. Under this condition, the simulations are performed solving Equations (16) and (17) using the alternate minimization algorithm described in Section 2.1 in a domain with the same dimensions shown in Figure $7(\mathrm{~b})$. The thickness of the sample is of the order of several millimeters, therefore, a plane-strain assumption is introduced.

The experimental and simulated force-displacement responses of the SENT sample are shown in Figure 9. After reaching the maximum force an abrupt failure occurs as the crack propagates across the sample. It can be seen that the SENT sample prior to failure, at about $1400 \mathrm{~N}$, follows a linear behavior. This brittle failure is common for PMMA and is attributed to the small fracture process zone. The reported sizes of fracture process zones are about $30 \square \mu \mathrm{m}^{29}$. The best agreement of the simulation with with the experiment is obtained with $\mathrm{G}_{\mathrm{c}}=418 \mathrm{Jm}^{-2}$ and $\mathrm{l}_{0}=36 \mu \mathrm{m}$ that satisfies the ratio $\frac{G_{I c}}{4 l_{0}}=2.9 \mathrm{MPa}$ obtained from the dog bone experiment. The damage length parameter compares favorably with the fracture process zone size for PMMA ${ }^{29}$. The Mode I stress intensity factor, $\mathrm{K}_{\mathrm{IC}}$, can be related to the critical energy release rate, $\mathrm{G}_{\mathrm{C}}$ as:

$$
G_{C}=\frac{K_{I C}^{2}}{E^{\prime}}
$$

where $E^{\prime}=E$ for the plane-stress condition and $E^{\prime}=E /\left(1-v^{2}\right)$ for the plane-strain condition. The value of $\mathrm{K}_{\mathrm{IC}}$ for PMMA is found to depend on the sample geometry and loading conditions ${ }^{31}$. Using Equation (37) a stress intensity factor of 1.07 MPa $\sqrt{m}$ is obtained, which is expected for the current geometry and quasi static crack propagation ${ }^{31}$. 


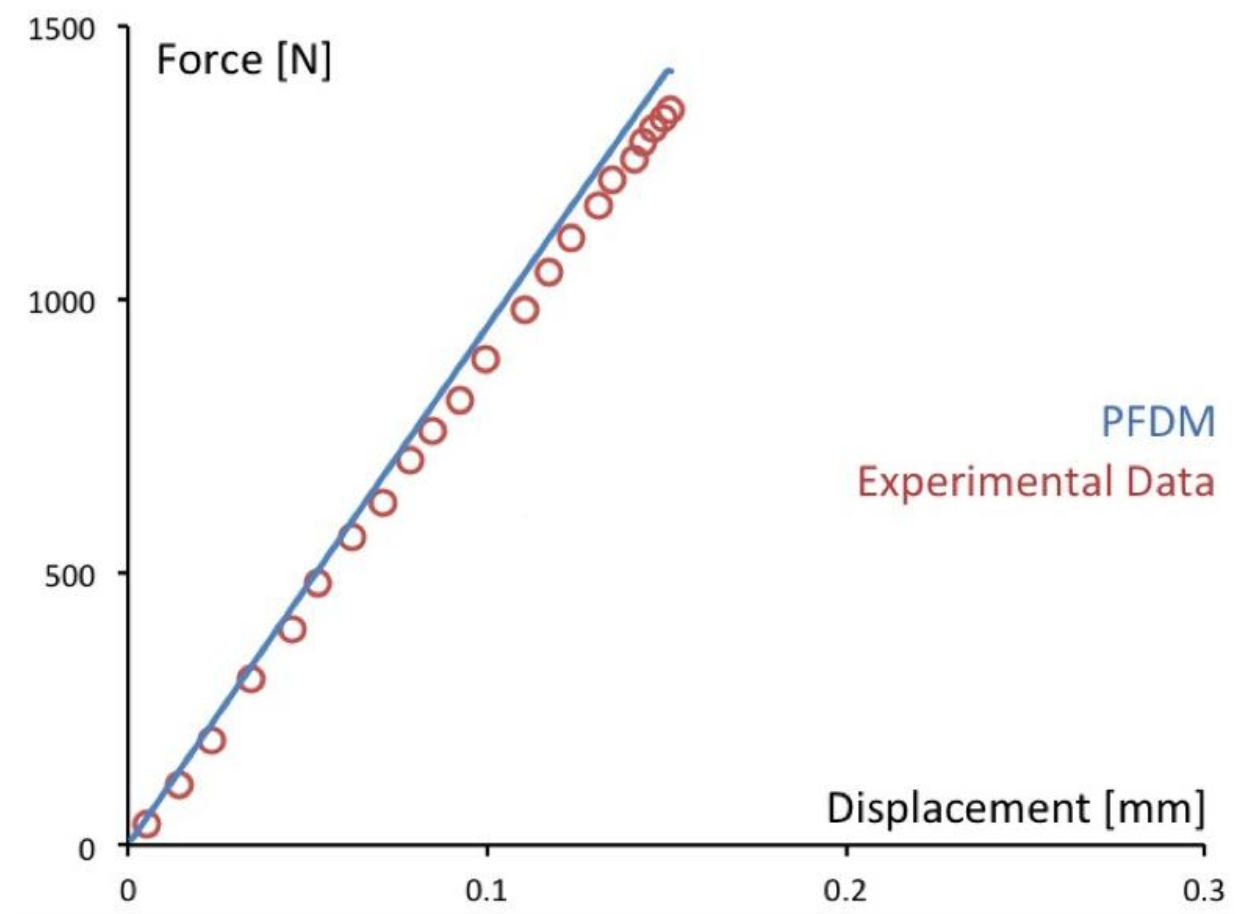

Figure 9: Force displacement curve for the SENT experiment and PFDM simulations with $G_{c}=418 \mathrm{Jm}^{-2}$ and $I_{0}=36 \mu \mathrm{m}$.

\section{Yield surfaces under biaxial loading}

In this section PFDM simulations are performed to predict the yield surfaces of several configurations used in the experiments shown in Figure 1. The elastic constants and PFDM parameters used in the simulations are obtained by the calibration in Section 3 and shown in Table 1.

\begin{tabular}{|c|c|}
\hline$E$ & $2483[\mathrm{MPa}]$ \\
\hline$\nu$ & 0.33 \\
\hline$\lambda=\frac{E v}{(1+v)(1-2 v)}$ & $1812[\mathrm{MPa}]$ \\
\hline$\mu=\frac{E}{2(1+v)}$ & $933[\mathrm{MPa}]$ \\
\hline$G_{c}$ & $418\left[\mathrm{Jm}^{-2}\right]$ \\
\hline$l_{0}$ & $36[\mu \mathrm{m}]$ \\
\hline
\end{tabular}

Table 1: Elastic constants, fracture toughness and length scale parameter calibrated by the experiments in Section 3. 


\subsection{Homogeneous deformation}

In the experiments of Bowden et al. ${ }^{2}$ a biaxial load is applied to a PMMA sample with thickness $1.5 \cdot 10^{-3} \mathrm{~m}$ and width $3.75 \cdot 10^{-2} \mathrm{~m}$. The authors divide the failure modes of the specimen into two types, ductile yielding and brittle fracture. The first one is identified by a stress drop in the stress-strain curve. While shear zones in the sample start forming from the edge of the sample due to the scratches on the surface, at yield the shear zones reach the central region and are widely spread over the whole sample and therefore, the experiment can be approximated as going through a state of homogeneous deformation.

To reproduce this experimental loading condition a sample under biaxial deformation is simulated with the PFDM. Plane strain condition is used following the assumptions in Bowden et al. ${ }^{2}$. The applied strain is

$\varepsilon(t)=\left[\begin{array}{ccc}\varepsilon_{11}(t) & 0 & 0 \\ 0 & \varepsilon_{22}(t) & 0 \\ 0 & 0 & 0\end{array}\right]$

Figure 10 shows the stress components at yield from the experiment of Bowden et $a l^{2}$, and the PFDM simulations. It is important to emphasize that the PFDM simulations are performed using the elastic constants in Table 1 without calibration against the experiments of Bowden et al. ${ }^{2}$.

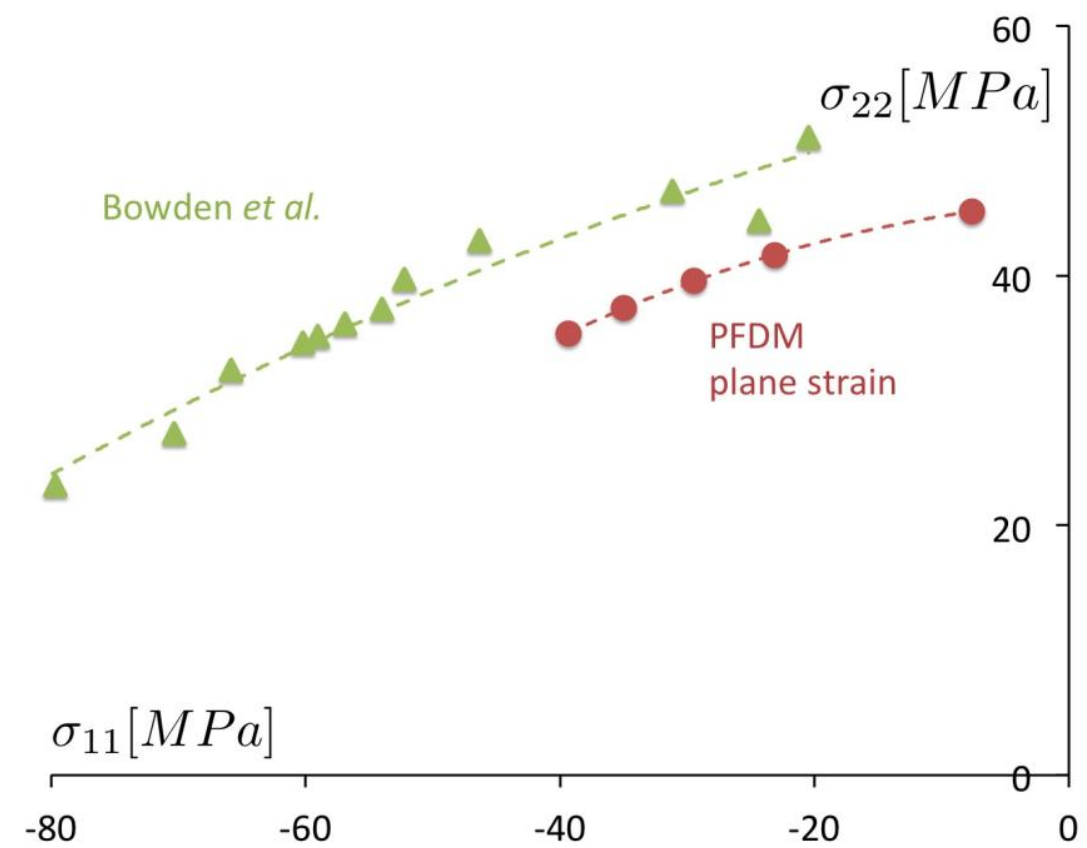

Figure 10: Stress components at yield from the experiment of Bowden et al. ${ }^{2}$, and the PFDM simulations. 
Figure 11 shows the stress invariants at failure for the data in Figure 10. In addition, the stress invariants at failure for a triaxial loading condition represented by Equation (36) and the model of Argon ${ }^{5}$ are shown. The experiments and the planestrain PFDM simulations follow a pressure modified von Mises law with slopes reported in Figure 11. The 3D PFDM simulations show a non linear relation with the deviatoric stress decreasing as the volumetric stress increases in agreement with MD simulations ${ }^{8}$. The results of Argon ${ }^{5}$ correspond to the local values of stress invariants at failure, therefore and they also follow a pressure modified von Mises relation. It should be noted that the PFDM does not predict yielding when the volumetric stress is below approximately -10MPa.

In Figure 1 when the volumetric stress is above $20 \mathrm{MPa}$ the yield surfaces do not agree with the pressure modified von Mises model. To explain this disagreement failure in a sample with a circular void is studied in the next section to incorporate the effect of defects that may drive failure under positive dilational stress.

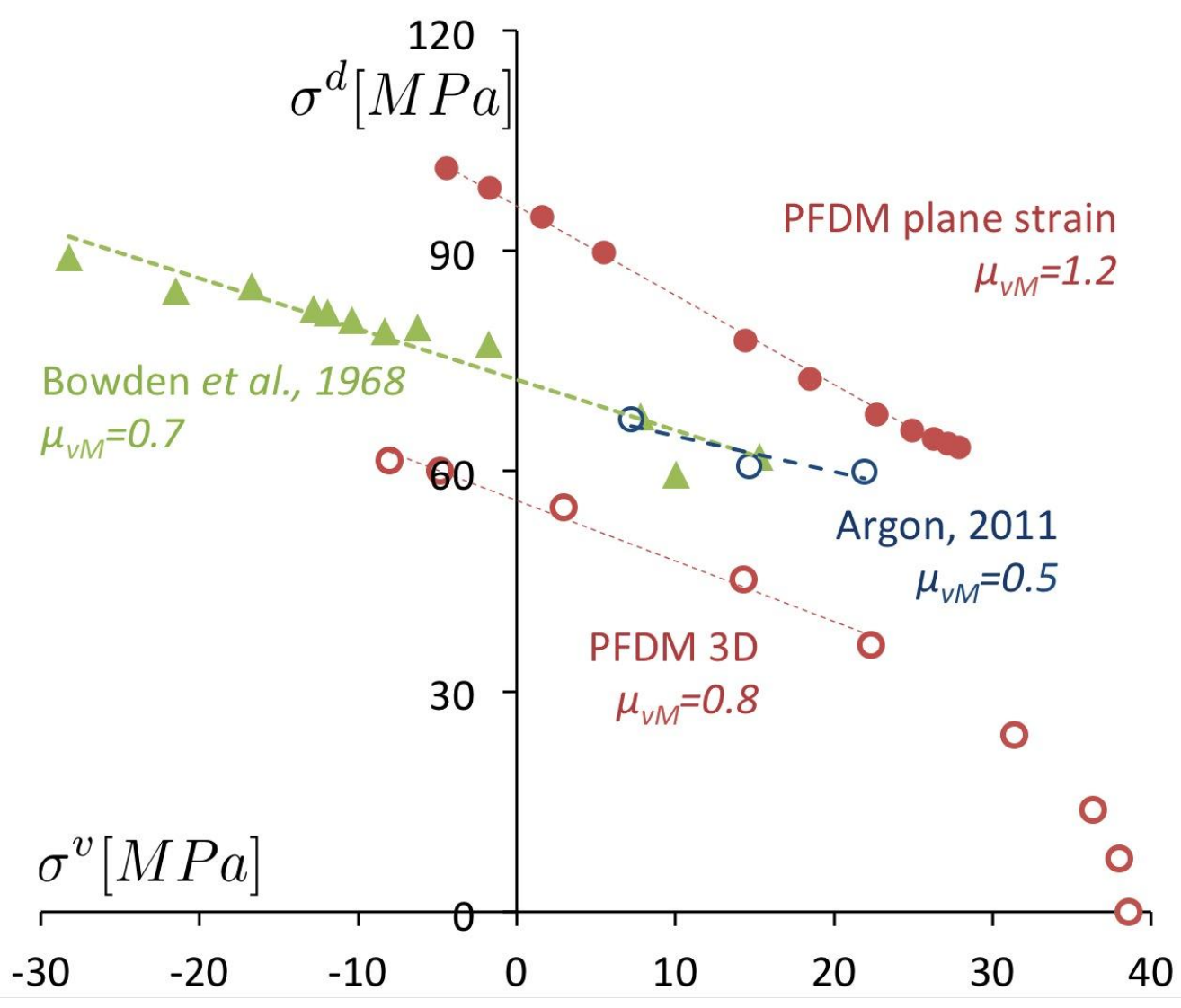


Figure 11: Deviatoric stress versus volumetric stress at failure from the experiment of Bowden et al. ${ }^{2}$, and Argon ${ }^{5}$ compared to PFDM simulations with plane strain condition (solid circles) and 3D deformation (open circles).

\subsection{Crazing induced by local failure at voids}

It is well established that crazing is a mode of deformation that develops macroscopic porosity 4, 5, 32-35 when a sample is deformed under volumetric loading. It is also recognized that that crazing occurs at stress concentrations produced by voids or surface grooves. Voids grow and coalesce to create crazes that lead to failure. Even though voids may not be present in the polymer before deformation the ultimate failure is driven by the deformation of these voids $4,5,34$. Here the focus is not on the nucleation of the voids but on how their presence affects the effective stresses at yield measured in experiments.

To this end we perform simulations with biaxial loading conditions reproducing the experiments by Sternstein6. In these experiments a biaxial tension is applied to a thin walled cylinder. Since the stress in the axial direction of the cylinder is larger crazes form in the direction perpendicular to this axis.

To study the local stress in the vicinity of the voids two different loading conditions are simulated with the geometries shown in Figure 12. In Figure 12(a) the thin wall assumption from Sternstain's experiments is used. Therefore, a biaxial displacement in the plane $x-y$ is applied under plane-stress conditions with a displacement $u_{0}$ in the $x$ direction and a displacement $z_{0}$ in the $y$ direction with $0.25<\gamma<1.00$. The second loading geometry is shown in Figure 12(b) where a displacement $u_{0}$ is applied in the $y$ direction and a displacement $\beta u_{0}$ in the $z$ direction with $0.15<\beta<0.40$ in a sample with finite thickness. These configurations are identified as "in-plane loading" and "transverse loading" respectively in the following. 


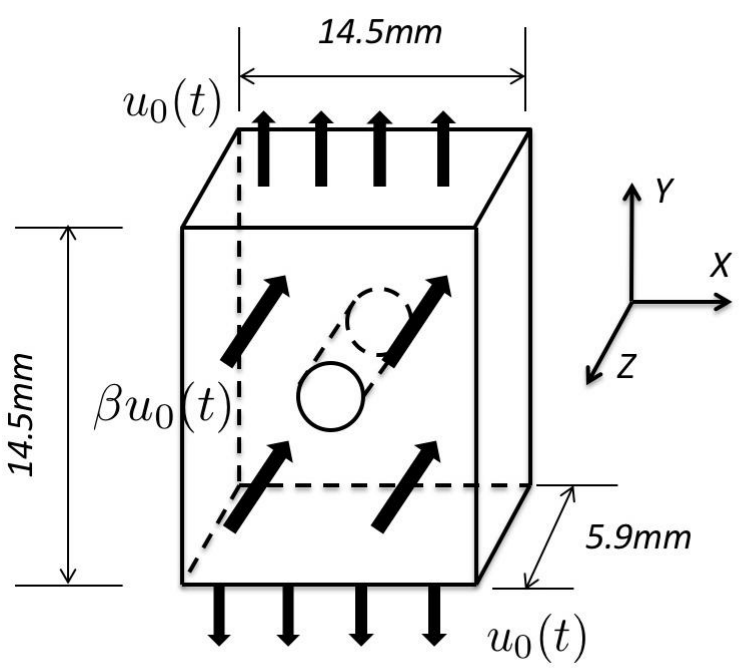

(a)

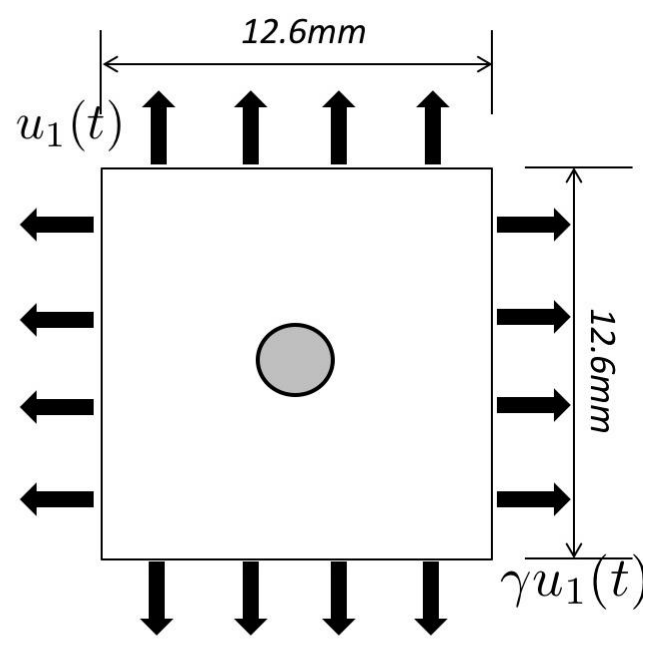

(b)

Figure 12: Loading and boundary conditions used in the PFDM simulations (a) in-plane loading and (b) transverse loading.

The diameter of the void is $1.5 \mathrm{~mm}$ in both simulations. With this geometry, the stress concentration factor in the elastic solution for the geometry shown in Figure 12 (a) is independent of the void $s^{2}{ }^{36}$. The value and location of the stress concentration factor for the problem in Figure 12(b) depends on the sample thickness and Poisson's ratio 373839 .

PFDM simulations are performed with both configurations and the yield condition is defined as the maximum of the rate of the mechanical work per unit volume. Figure 13 and 14 show contour plots of the damage field, in both cases the craze grows in the direction perpendicular to the maximum principal stress. 


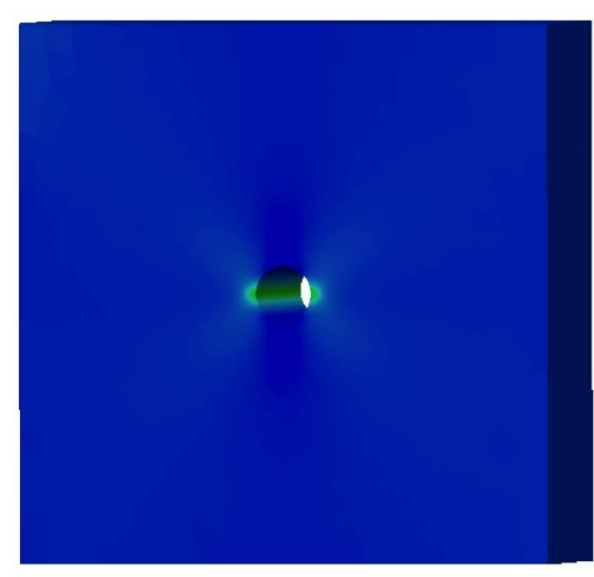

(a)

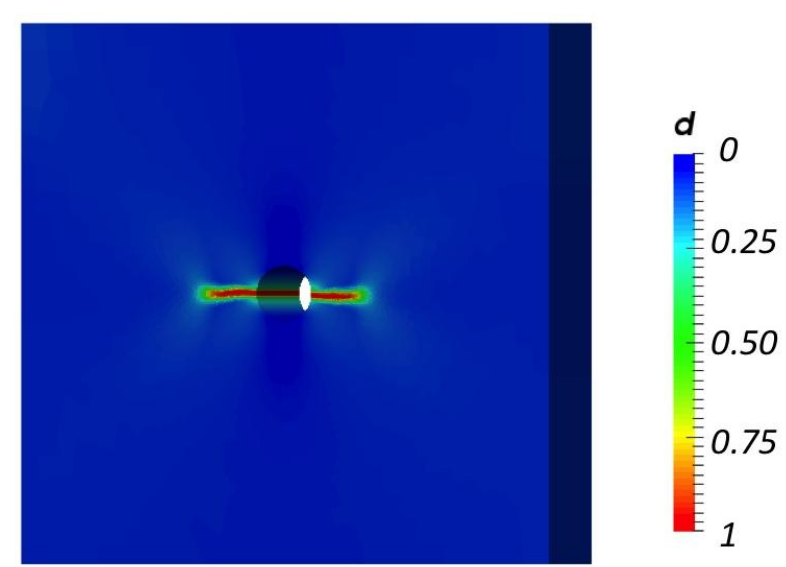

(b)

Figure 13: Contour plots of the damage field for the transverse loading condition with $\beta=-0.1$ (a) $u_{0}=1.058 \cdot 10^{-4} \mathrm{~m}$ and (b) $u_{0}=1.062 \cdot 10^{-4} \mathrm{~m}$.

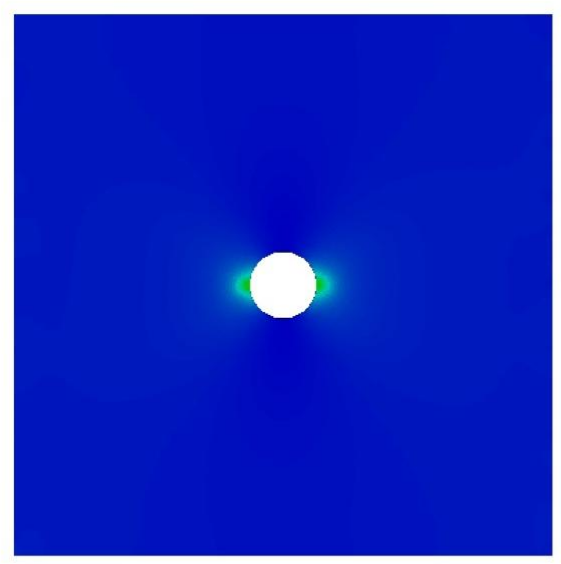

(a)

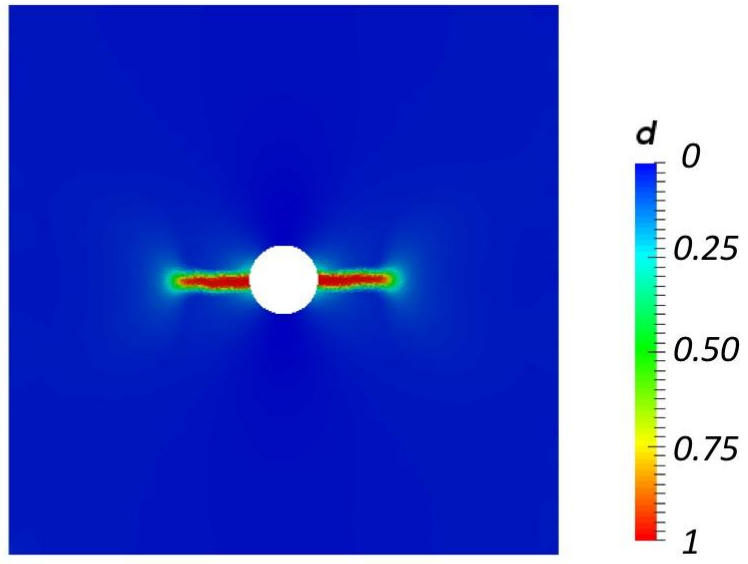

(b)

Figure 14: Contour plots of the damage field for the in-plane loading with $\gamma=0.25$ (a) $u_{0}=7.06 \cdot 10^{-5} \mathrm{~m}$ and (b) $u_{0}=7.08 \cdot 10^{-5} \mathrm{~m}$.

The yield surfaces are shown in Figure 15 and compared to the experimental results of Sternestein et al. ${ }^{3,6}$ and Argon et al. ${ }^{4}$. Both experiments report the applied stress at which the onset of crazing is observed.

When the volumetric stress is below $10 \mathrm{MPa}$ the relation between the deviatoric stress and the volumetric stress remains linear even in the presence of a void but with a larger slope than previously estimated. When the volumetric stress is larger than 10Mpa the yield surfaces obtained with the PFDM have similar trends to the 
experiments of Sternstein et al. ${ }^{6}$. These findings explain what seems a contradictory result in the yield surface proposed in the work of Sternstein et al. 3,6, in which the yield surface depends on the principal stresses, and the volumetric stress as:

$\left|\begin{array}{ll}1 & 2\end{array}\right|=A+\frac{B}{v}$ (39)

where A and B are two temperature dependent parameters that are calculated by fitting to experimental results.

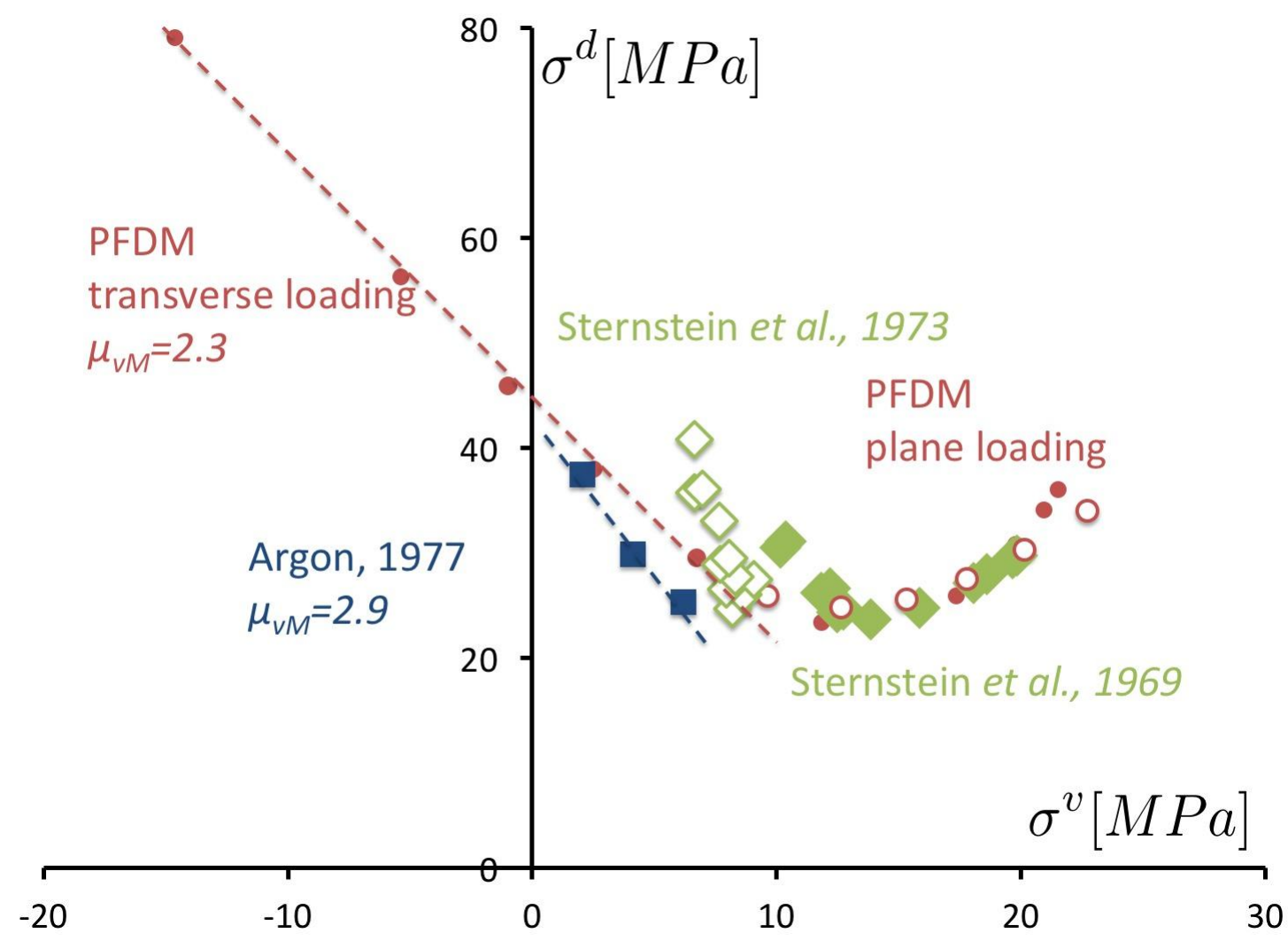

Figure 15: Applied deviatoric stress versus volumetric stress at failure from Argon et al. ${ }^{4}$ and Sternstein et al. ${ }^{3,6}$ experiments and PFDM with plane loading (open circles) and transverse loading (solid circles).

At first, it seems that the results in Figure 15 contradict the pressure modified von Misses criterion in which the deviatoric stress decreases as the volumetric stress increases. To explain this difference, it is necessary to consider the value of the local stresses at failure. To this end, the closed form solution of elastic problem of an elliptical hole in an infinite plate shown in Figure 16(a) is considered. The applied stress is $\sigma$ in the $x$ direction and $\gamma \sigma$ in the $y$ direction with $-0.9<\gamma<0.9$ and $\sigma>0$. The maximum stress component occurs is in the $y$ direction in the periphery of the hole 
along the largest axis of the hole. The value can be calculated from Inglis ${ }^{37}$ equations as:

$$
\sigma_{22}^{\text {local }}=\sigma\left(f_{s}-\gamma\right)
$$

with $f_{s}=\left(1+2 \frac{a}{b}\right)$. To obtain the value of the local stress at failure a pressure modified von Mises criterion (Equation (1)) is used. The stress invariants are calculated using Equations (2) and (3) with $\sigma_{11}=\sigma_{33}=0$ and $\sigma_{22}=\sigma_{22}$ local, this results the following condition for the applied stress at yield:

$$
\sigma=\frac{3 \sigma_{\text {crit }}}{\left(f_{s}-\gamma\right)\left(3+\mu_{v M}\right)}
$$

It is important to note that any other local failure criteria such as the ones proposed by Argon ${ }^{5}$ or by Xie et al. ${ }^{28}$ would lead to an identical relation between $\gamma$ and $\sigma$, but with different factors. The applied stress invariants at yield from Equation (41) result:

$$
\begin{array}{r}
\sigma^{v o l}=\frac{(\gamma+1) \sigma_{c r i t}}{\left(f_{s}-\gamma\right)\left(3+\mu_{v M}\right)}(42) \\
\sigma^{d e v}=\frac{\sigma_{c r i t}}{\left(f_{s}-\gamma\right)\left(3+\mu_{v M}\right)} \sqrt{\frac{\left(\gamma^{2}-\gamma+1\right)}{2}}
\end{array}
$$

In Figure 16(b) the stress invariants $\sigma^{v o l}$ and $\sigma^{d e v}$ are calculated with Equations (42) and (43) and a value $\frac{\sigma_{c r i t}}{\left(3+\mu_{v M}\right)}=100 M P a$. The parameter $f_{s}=3$ corresponds to a circular void while $f_{s}=5$ or 10 represents crack like geometries. The minimum of the deviatoric in Figure 16(b) stress occurs for

$$
\gamma=\frac{\left(f_{s}-2\right)}{\left(2 f_{s}-1\right)}
$$

Replacing Equation (44) in Equation (42), the critical volumetric stress at which the deviatoric stress starts growing reduces to

$$
\sigma_{c}^{v o l}=\frac{\left(f_{s}-1\right) \sigma_{c r i t}}{\left(f_{s}^{2}-f_{S}+\gamma\right)\left(3+\mu_{v M}\right)}
$$

Therefore, under the simplifying assumptions in this model the critical volumetric stress at which the pressure modified von Misses relation breaks down decreases as the voids have more crack like shape (larger $f_{s}$ ). 


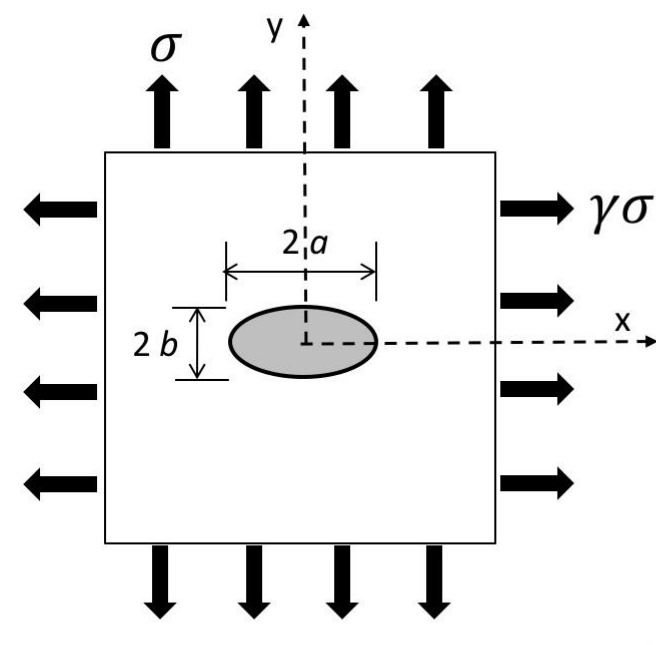

(a)

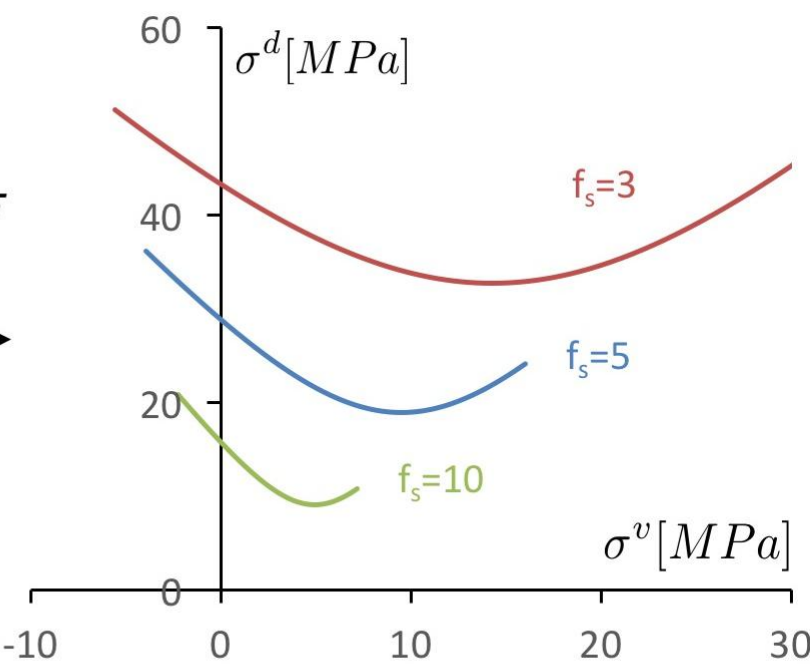

(b)

Figure 16: (a) Geometry used in the elastic solution of an infinite plate with an elliptical void. (b) Yield surface obtained from the elastic solution.

\section{Summary}

PFDM simulations were performed to calculate the yield surface of PMMA over a range of volumetric stresses from -30Mpa to $30 \mathrm{Mpa}$. The elastic constants and two parameters that represent the fracture toughness and a characteristic length scale were calibrated with a simple tension and a fracture toughness experiments.

The damage model proposed by Miehe et al. ${ }^{19}$ that takes into account the sign of the principal strain direction for failure was chosen to simulate failure of PMMA under several loading conditions. The PFDM simulations under compressive volumetric stress are used to obtain the yield surface for shear yielding. In this case the yield surface follows a pressure modified von Mises relation in agreement with experiments 1,2. The pressure modified linear relation breaks down in the experiments that report crazing when the volumetric stress exceeds $10 \mathrm{Mpa}{ }^{6}$. To understand the differences among these experimental results simulations with a geometry containing a void under two different loading conditions were performed. With this configuration the slope of the yield surface changes when the volumetric applied stresses are above $10 \mathrm{Mpa}$ and the pressure modified von Mises relation does not hold. Contrary to the von Mises relation the applied deviatoric and volumetric stresses increase at failure. It is important to emphasize that these relation holds only for the applied stresses and should not be used as a local yield criteria. 
In summary, the PFDM simulations show that a single phase field model can be used to describe both the onset of shear yielding and crazing when the appropriate geometry is considered. While the local failure behavior can be modeled with the same constitutive equations during these processes the linear relationship between the applied deviatoric and volumetric stresses at failure breaks down. The differences in the yield surface for shear yielding (negative volumetric stress) and crazing (positive volumetric stress) can be explained by the presence of voids and other defects that localize failure modifying the relationship between the applied stress invariants. Furthermore, a simplified model with the elastic solution of an elliptical void in an infinite plate shows that even though the local stress invariants follow a pressure modified von-Mises at yield, this is not true for the applied stress invariants that are reported in experiments if voids, groves or other defects are present in the sample.

\section{Acknowledgements}

This work was supported by the US National Science Foundation, CMMI Grant No. 0826356 and from the Boeing Co. Discussions with Dr. Christensen, Dr. Gosse and Prof. Strachan are greatly acknowledged.

\section{References}

1. Quinson, R., Perez, J., Rink, M. \& Pavan, A. Yield criteria for amorphous glassy polymers. Journal of Materials Science 32, 1371-1379 (1997).

2. Bowden, P. B. \& Jukes, J. A. The plastic yield behaviour of polymethylmethacrylate. Journal of Materials Science 3, 183-190 (1968).

3. Sternstein, S. S. \& Myers, F. A. Yielding of glassy polymers in the second quadrant of principal stress space. J. of Macromolecular Sc., Part B 8, 539-571 (1973).

4. Argon, A. S. \& Hannoosh, J. G. Initiation of crazes in polystyrene. Philosophical Magazine 36, 1195-1216 (1977).

5. Argon, A. S. Craze initiation in glassy polymers. Polymer 52, 2319-2327 (2011).

6. Sternstein, S. S. \& Ongchin, L. Yield criteria for plastic deformation of glassy high polymers in general stress fields. Polymer preprints 10, 1117-1124 (1969).

7. Mahajan, D. K. \& Hartmaier, A. Mechanisms of crazing in glassy polymers revealed by molecular dynamics simulations. Phys. Rev. E 86, 021802 (2012).

8. Jaramillo, E., Wilson, N., Christensen, S., Gosse, J. H. \& Strachan, A. Energybased yield criterion for PMMA from large-scale molecular dynamics simulations. Phys. Rev. B 85, 024114 (2012).

9. Estevez, R., Tijssens, M. G. A. \& Van der Giessen, E. Modeling of the 
competition between shear yielding and crazing in glassy polymers. Journal of the Mechanics and Physics of Solids 48, 2586-2617 (2000).

10. Tijssens, M. G. A., Van der Giessen, E. \& Sluys, L. J. Modeling of crazing using a cohesive surface methodology. Mechanics of Materials 32, 19-35 (2000).

11. Seelig, T. \& Van der Giessen, E. A cell model study of crazing and matrix plasticity in rubber-toughened glassy polymers. Computational Materials Science 45, 725-728 (2009).

12. Ponson, L. \& Bonamy, D. Crack propagation in brittle heterogeneous solids: Material disorder and crack dynamics. International Journal of Fracture 162, 21-31 (2010).

13. Heyden, S., Li, B., Weinberg, K., Conti, S. \& Ortiz, M. A micromechanical damage and fracture model for polymers based on fractional strain-gradient elasticity. Journal of the Mechanics and Physics of Solids 74, 175-195 (2015).

14. Totry, E., González, C. \& LLorca, J. Prediction of the failure locus of C/PEEK composites under transverse compression and longitudinal shear through computational micromechanics. Composites Science and Technology 68, 31283136 (2008).

15. Mendoza-Jasso, A. J., Goodsell, J. E., Ritchey, A. J. \& Pipes, R. B. A parametric study of fiber volume fraction distribution on the failure initiation location in open hole off-axis tensile specimen. Composites Science and Technology 71, 1819-1825 (2011).

16. Tran, T. D., Kelly, D., Prusty, B. G., Gosse, J. H. \& Christensen, S. Micromechanical modelling for onset of distortional matrix damage of fiber reinforced composite materials. Composite Structures 94, 745-757 (2012).

17. Francfort, G. A. \& Marigo, J.-J. Revisiting brittle fracture as an energy minimization problem. Journal of the Mechanics and Physics of Solids 46, 1319-1342 (1998).

18. Amor, H., Marigo, J.-J. \& Maurini, C. Regularized formulation of the variational brittle fracture with unilateral contact: Numerical experiments. Journal of the Mechanics and Physics of Solids 57, 1209-1229 (2009).

19. Miehe, C., Hofacker, M. \& Welschinger, F. A phase field model for rateindependent crack propagation: Robust algorithmic implementation based on operator splits. Comput. Methods Appl. Mech. Engrg. 199, 2765-2778 (2010).

20. Pandolfi, A. \& Ortiz, M. An eigenerosion approach to brittle fracture. Int. J. Numer. Meth. Engng. 92, 694-714 (2012).

21. Hesch, C. \& Weinberg, K. Thermodynamically consistent algorithms for a finite-deformation phase-field approach to fracture. Int. J. Numer. Meth. Engng. 99, 906-924 (2014).

22. Bourdin, B., Francfort, G. A. \& Marigo, J.-J. The Variational Approach to Fracture. Journal of Elasticity 91, 5-148 (2008).

23. Clayton, J. D. \& Knap, J. A geometrically nonlinear phase field theory of brittle fracture. International Journal of Fracture 189, 139-148 (2014).

24. Borden, M. J., Verhoosel, C. V., Scott, M. A., Hughes, T. J. R. \& Landis, C. M. A phase-field description of dynamic brittle fracture. Comput. Methods Appl. Mech. Engrg. 217-220, 77-95 (2012).

25. Hossain, M. Z., Hsueh, C. J., Bourdin, B. \& Bhattacharya, K. Effective toughness 
of heterogeneous media. Journal of the Mechanics and Physics of Solids 71, 1532 (2014).

26. Schänzel, L.-M. Phase field modeling of fracture in rubbery and glassy polymers at finite thermo-viscoelastic deformations. (PHD Thesis, University of Stuttgart, 2015).

27. Bourdin, B., Francfort, G. A. \& Marigo, J.-J. Numerical experiments in revisited brittle fracture. Journal of the Mechanics and Physics of Solids 48, 797-826 (2000).

28. Xie, Y., Mao, Y., Sun, L. \& Koslowski, M. Local versus average field failure criterion in amorphous polymers. Modelling and Simulation in Materials Science and Engineering 23, 025004 (2015).

29. Williams, J. G. Fracture Mechancis of Polymer. (Ellis Horwood Limited, 1984).

30. ASTM D5045-14. Standard Test Methods for Plane-Strain Fracture Toughness and Strain Energy Release Rate of Plastic Materials. (ASTM International, 2014). at <www.astm.org>

31. Kravchenko, S. G., Kravchenko, O. G. \& Sun, C.-T. A two-parameter fracture mechanics model for fatigue crack growth in brittle materials. Engineering Fracture Mechanics 119, 132-147 (2014).

32. Kramer, E. J. Microscopic and molecular fundamentals of crazing. 1-56 (Springer, 1983).

33. Kramer, E. J. \& Berger, L. L. Fundamental processes of craze growth and fracture. Crazing in Polymers Vol 2 91/92, 1-68 (1990).

34. Basu, S., Mahajan, D. K. \& Van der Giessen, E. Micromechanics of the growth of a craze fibril in glassy polymers. Polymer 46, 7504-7518 (2005).

35. Arzhakova, O. V., Dolgova, A. A., Yarysheva, L. M., Volynskii, A. L. \& Bakeev, N. F. Specific features of the environmental crazing of poly ethylene terephthalate fibers. Polymer 56, 256-262 (2015).

36. Timoshenko, S. \& Goodier, J. N. Theory of Elasticity, McGraw Hill, New York USA (1970).

37. Inglis, C. E. Stresses in Plates Due to the Presence of Cracks and Sharp Corners. Transactions of the Institute of Naval Architects 55, 219-241 (1913). 